\title{
Accurate Evaluation of Finite Laguerre Series
}

\author{
Keshan He ${ }^{1, a, *}$, Peibing Du ${ }^{1, a}$, Hao Jiang ${ }^{1, a}$, Xueci Zhao ${ }^{1, a}$, Housen $\mathrm{Li}^{3, \mathrm{~b}}$ and Lizhi Cheng, \\ ${ }^{1}$ School of Science, National University of Defense Technology, Changsha, China \\ ${ }^{2}$ College of Computer, National University of Defense Technology, Changsha, China \\ ${ }^{3}$ Max Planck Institute for Biophysical Chemistry, Am Fassberg 11, 37077 Geottingen, Germany \\ a $\{$ hekeshan11, dupeibing10, zhaoxueci11, Hao Jiang, Lizhi Cheng\}@nudt.edu.cn, \\ b housen.li@mplibpc.mpg.del, \\ *corresponding author
}

Keywords: Compensated Algorithm, Laguerre Series, Clenshaw, Floating-Point Arithmetic, Round-off Error

\begin{abstract}
A compensated algorithm is presented to evaluate finite Laguerre series. We record round-off error in Clenshaw algorithm by using error-free transformations (EFTs). In this work, we establish a valid error estimate that is as accurate as the Clenshaw scheme using twice the working precision. Numerical experiments illustrate that our compensated algorithm is more accurate than the Clenshaw algorithm and faster than the DDClenshaw algorithm (Clenshaw in double-double arithmetic).
\end{abstract}

\section{Introduction}

The Generalized Laguerre polynomial is widely used in numerical analysis [1, 2, 3] and Quantum [4]. It is defined with three-term recurrence relation as follow.

$$
\left\{\begin{array}{l}
L_{0}^{(\alpha)}(x)=1 \\
L_{1}^{(\alpha)}(x)=1+\alpha-x, \\
L_{k+1}^{(\alpha)}(x)=\frac{-x+2 k+1+\alpha}{k+1} L_{k}^{(\alpha)}(x)-\frac{k+\alpha}{k+1} L_{k-1}^{(\alpha)}(x) .
\end{array}\right.
$$

The Laguerre polynomials are orthogonal polynomials with respect to an inner product $<f, g>=\int_{0}^{+\infty} f(x) g(x) e^{-x} d x$. The polynomial represented in Laguerre basis is $p(x)=\sum_{j=0}^{n} a_{j} L_{j}^{(\alpha)}(x)$ where coefficients $a_{j}$ are floating-point numbers.

Clenshaw Algorithm [5] is a recursive method to calculate a linear combination of Chebyshev polynomials. It can be generalized to any class of function which can be defined by a three-term recurrence relation. The relative error bound proved in literatures $[6,7]$ is

$$
\frac{|p(x)-\hat{p}(x)|}{|p(x)|} \leq \operatorname{cond}(p, x) \times o(u),
$$


where $\hat{p}(x)$ is the result of Clenshaw algorithm in floating-point arithmetic. When the problem is ill-conditioned, numerous compensated algorithms $[8,9,10]$ based on error-free transformations [11] are proposed to evaluate different polynomials.

We propose a compensated algorithm to evaluate Laguerre series based on error-free transformation. The algorithm can obtain a more accurate result by compensating the round-off errors to the original result. We demonstrate that the forward relative error bound satisfies

$$
\frac{|p(x)-\hat{p}(x)|}{p(x)} \leq o(u)+\operatorname{cond}(p, x) \times o\left(u^{2}\right) .
$$

This paper is organized as follows. We introduce basic preliminaries and notations in Section 2, including error-free transformations and Clenshaw algorithm. In Section 3, we present the compensated Clenshaw algorithm and analyze the forward error of Clenshaw and Compensated Clenshaw algorithm. In Section 4, we show the experimental results to indicate our algorithm is efficient and accurate.

\section{Preliminaries and Notations}

\subsection{Basic Definitions and Notation}

Throughout the paper we assume a floating-point arithmetic adhering to IEEE 754 floating-point standard [12] and no overflow nor underflow occurs. We also assume that $f l(\cdot)$ is the result of a floating-point computation. Here, the computations are produced in a floating-point arithmetic which obeys the models.

$$
f l(x \text { op } y)=(x \text { op } y)(1+\rho)=\frac{(x \text { op } y)}{1+\alpha}, \quad|\rho|,|\alpha| \leq u,
$$

where op $\in\{+,-, \times, /\}$ and $u$ is the working precision. Besides, we note $\gamma_{n}:=n u /(1-n u)=n u+\mathcal{O}\left(u^{2}\right)$ and we use $\hat{a}$ to represent the computed element of a in floatingpoint arithmetic. Finally, an elementary classical result of error analysis states that if $\left|\delta_{i}\right| \leq u$ then $\prod_{i=1}^{n}\left(1+\delta_{i}\right)=1+\theta_{n}$. with $\theta_{n} \leq \gamma_{n}$. $\prod_{i=1}^{n}\left(1+\delta_{i}\right)=1+\theta_{n}$

\subsection{Error-free Transformations}

In this subsection we review well-known results called error-free transformations (EFTs) . Given $a, b \in \mathbb{F}, x=f l(a$ op $b) \in \mathbb{F}$, then exist $y$ such that $a$ op $b=x+y$.

We show algorithms 1-3 about addition and product of two floating-point numbers proposed respectively by Knuth [13] and Dekker [14], respectively.
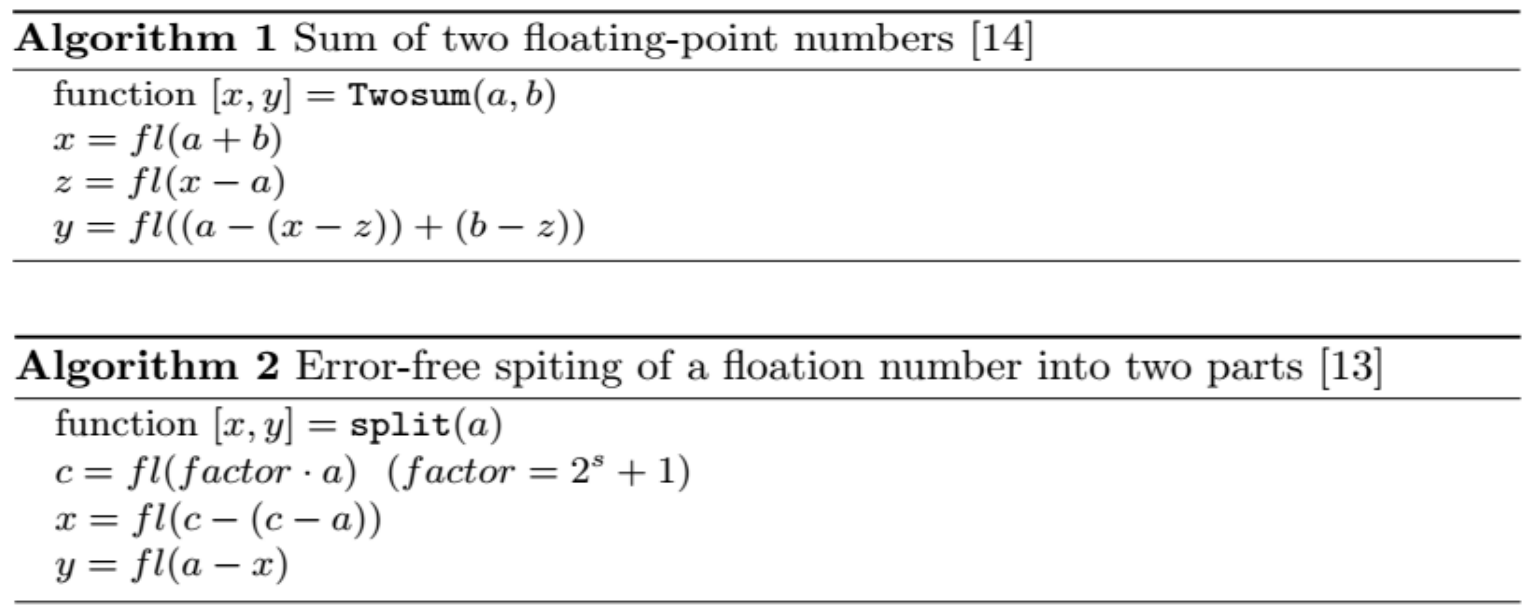

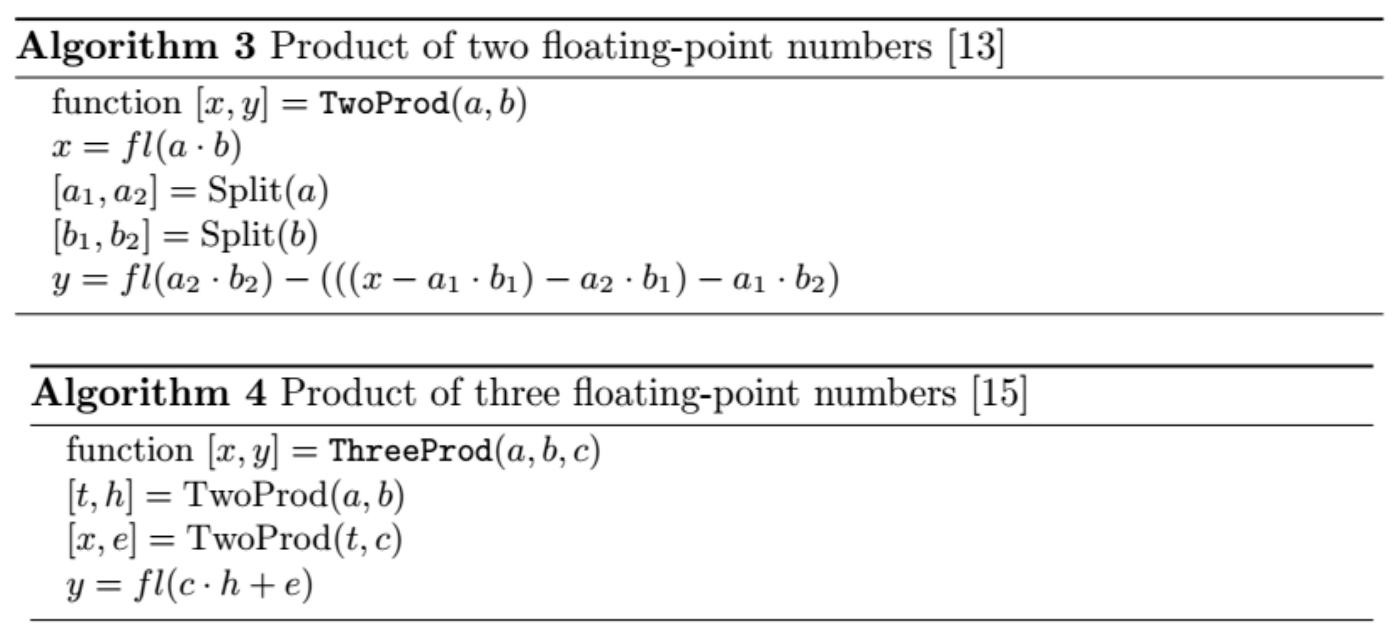

A compensated algorithm [15] for product of three floating-point numbers in Algorithm 4 is also presented. There are three properties of ThreeProd which will be used in error analysis:

$$
\begin{aligned}
& \text { 1. }|e| \leq u|a b c|+u|h c| \leq u|a b c|+u^{2}|a b c| ; \\
& \text { 2. }|c h+e| \leq|c h|+|e| \leq 2 u|a b c|+u^{2}|a b c| ;
\end{aligned}
$$

3. If $x+y=a b c-\tau$ in ThreeProd algorithm, then

$$
|\tau| \leq \gamma_{2} \gamma_{6}|a b c|, \quad|y| \leq 2 u|a b c|+14 u^{2}|a b c| \text {. }
$$

\subsection{Clenshaw Algorithm and Condition Number}

The Clenshaw algorithm [5] of generalized Laguerre polynomial shows in Algorithm 5.

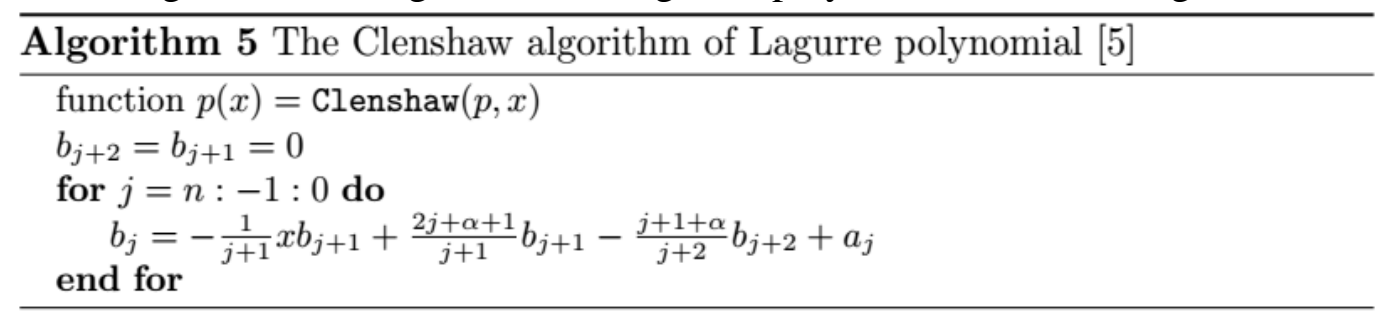

Reviewing [16], their works put forward a general condition number for polynomial basis defined by linear recurrence. Before giving the condition number of Laguerre polynomial, we show an absolute polynomial.

Definition 1. Let $L_{k}(x)$ be a Laguerre polynomial. The absolute polynomial $\tilde{L}_{k}^{(\alpha)}(x)$ can be defined as follow.

$$
\tilde{L}_{k}^{(\alpha)}(x)=\frac{x+2 k+\alpha+1}{k+1} \tilde{L}_{k-1}^{(\alpha)}(x)+\frac{k+\alpha}{k+1} \tilde{L}_{k-2}^{(\alpha)}(x),
$$

where $\tilde{L}_{\mathrm{n}}^{(\alpha)}=1, \tilde{L}_{1}^{(\alpha)}=1+\alpha+x$ and $\left|L_{v}^{(\alpha)}(x)\right| \leq \tilde{L}_{v}^{(a)}(x)$, for all $x \geq 0$.

Motivated by $[10,11,15]$, we show the condition number of Laguerre polynomial series.

Definition 2. Let $p(x)=\sum_{i=n}^{n} a_{k} L_{v}^{(\alpha)}(x)$, the relative condition number is

$$
\operatorname{cond}(p, x)=\frac{\tilde{p}(x)}{|p(x)|}=\frac{\sum_{i=0}^{n}\left|a_{k}\right| \tilde{L}_{k}^{(\alpha)}(|x|)}{|p(x)|},
$$

where $L_{v}^{(\alpha)}(x)$ is Laguerre polynomial, $\hat{L}_{v}^{(\alpha)}(x)$ is absolute polynomial. 


\section{Compensated Algorithm and Error Analysis}

\subsection{Clenshaw Algorithm and Condition Number}

In this section, we analyze the round-off error of Clenshaw algorithm and present a compensated Clenshaw algorithm to compute finite Laguerre series accurately. Furthermore, we exhibit its error bound.

Previously, we divide all real coefficients into three parts as following form in order to analyze errors.

Where $A^{(h)}, A^{(l)} \in \mathbb{F}, A^{(m)} \in \mathbb{R}, A^{(m)} \leq u A^{(l)}, A^{(l)} \leq u A^{(h)}$

$$
A=A^{(h)}+A^{(l)}+A^{(m)}
$$

Actually, in floating-point arithmetic, round-off error is almost produced in each addition, subtraction or multiplication in Clenshaw algorithm. We choose the recurrence relation at $i$ th step of Clenshaw Algorithm for analysis, we have

$$
\hat{b}_{j}=\left(A_{j}^{(h)} \otimes x \oplus B_{j}^{(h)}\right) \otimes b_{j+1} \oplus C_{j+1}^{(h)} \otimes b_{j+2} \oplus a_{j} .
$$

As for theoretical computation related with (8), we deduce that

$$
\begin{aligned}
& b_{j}=\left(A_{j} x+B_{j}\right) b_{j+1}+C_{j+1} b_{j+2}+a_{j} \\
& =\left[\left(A_{j}^{(h)}+A_{j}^{(l)}+A_{j}^{(m)}\right) x+\left(B_{j}^{(h)}+B_{j}^{(l)}+B_{j}^{(m)}\right)\right] b_{j+1}+\left(C_{j+1}^{(h)}+C_{j+1}^{(l)}+C_{j+1}^{(m)}\right) b_{j+2}+a_{j} .
\end{aligned}
$$

In Algorithm 4 we use EFTs and ThreeProd to eliminate every error and obtain that

$$
\begin{array}{ll}
{\left[s_{j}, \alpha_{j}\right]=\operatorname{ThreeProd}\left(A_{j}^{(h)}, x, \hat{b}_{j+1}\right),} & {\left[t_{j}, \beta_{j}\right]=\operatorname{TwoProd}\left(B_{j}^{(h)}, \hat{b}_{j+1}\right),} \\
{\left[u_{j}, \xi_{j}\right]=\operatorname{TwoProd}\left(C_{j+1}^{(h)}, \hat{b}_{j+2}\right),} & {\left[v_{j}, \eta_{j}\right]=\operatorname{TwoSum}\left(s_{j}, t_{j}\right),} \\
{\left[w_{j}, \theta_{j}\right]=\operatorname{TwoSum}\left(v_{j}, u_{j}\right),} & {\left[\hat{b}_{j}, \zeta_{j}\right]=\operatorname{TwoSum}\left(w_{j}, a_{j}\right) .}
\end{array}
$$

Contrast with (9), the round-off error of method (8) is

$$
\begin{aligned}
& e_{j}=\left(A_{j}^{(l)}+A_{j}^{(m)}\right) x \hat{b}_{j+1}+\left(B_{j}^{(l)}+B_{j}^{(m)}\right) \hat{b}_{j+1}+\left(C_{j+1}^{(l)}+C_{j+1}^{(m)}\right) \hat{b}_{j+2}+\tau_{j}+\alpha_{j}+\beta_{j}+\xi_{j}+\eta_{j}+\theta_{j}+\zeta_{j} \text {. } \\
& \begin{array}{l}
\text { Algorithm 6 Compensate Clenshaw Algorithm } \\
\hline \text { function CompClenshaw }(p, x) \\
\text { Initialization the starting } \hat{b}_{n+2}=\hat{b}_{n+1}=0, \epsilon b_{n+2}=\epsilon b_{n+1}=0 \\
\text { for } j=n:-1: 0 \text { do }
\end{array} \\
& {\left[A_{j}^{(h)}, A_{j}^{(l)}\right]=\text { div_d_d }(-1, n+1)} \\
& {\left[B_{j}^{(h)}, B_{j}^{(l)}\right]=d i v \_d \_d(2 n+\alpha+1, n+1)} \\
& {\left[C_{j}^{(h)}, C_{j}^{(l)}\right]=d_{i v} d_{-} d(-n-1-\alpha, n+1)} \\
& {\left[s_{j}, \alpha_{j}\right]=\operatorname{ThreeProd}\left(A_{j}^{(h)}, x, \hat{b}_{j+1}\right)} \\
& {\left[t_{j}, \beta_{j}\right]=\operatorname{TwoProd}\left(B_{j}^{(h)}, \hat{b}_{j+1}\right)} \\
& {\left[u_{j}, \xi_{j}\right]=\operatorname{TwoProd}\left(C_{j+1}^{(h)}, \hat{b}_{j+2}\right)} \\
& {\left[v_{j}, \eta_{j}\right]=\operatorname{TwoSum}\left(s_{j}, t_{j}\right)} \\
& {\left[w_{j}, \theta_{j}\right]=\operatorname{TwoSum}\left(v_{j}, u_{j}\right)} \\
& {\left[\hat{b}_{j}, \zeta_{j}\right]=\operatorname{TwoSum}\left(w_{j}, a_{j}\right)} \\
& \hat{s}_{j}=\alpha_{j} \oplus \beta_{j} \oplus \xi_{j} \oplus \eta_{j} \oplus \theta_{j} \oplus \zeta_{j} \oplus\left(A_{j}^{(l)} \otimes x \otimes \hat{b}_{j+1} \oplus B_{j}^{(l)} \otimes \hat{b}_{j+1} \oplus C_{j+1}^{(l)} \otimes \hat{b}_{j+2}\right) \\
& \epsilon \hat{b}_{j}=A_{j}^{(h)} \otimes x \otimes \epsilon b_{j+1} \oplus C_{j+1}^{(h)} \otimes \epsilon \hat{b}_{j+2} \oplus \hat{s}_{j} \\
& \text { end for } \\
& r e s=\hat{b}_{0}+\epsilon \hat{b}_{0}
\end{aligned}
$$

Then we can obtain Theorem 1 as follow.

Theorem 1. Let $p(x)=\sum_{i=n}^{n} a_{k} L_{b}^{(\alpha)}(x) p(x)=\sum_{i=0}^{n} a_{i} L_{i}^{(\alpha)}(x), b_{0}$ be the computed result by Clenshaw algorithm in floating-point arithmetic, given $e_{i}=\tau_{i}+\alpha_{i}+\beta_{i}+\xi_{i}+\eta_{i}+\theta_{i}+\zeta_{i}, j=0, \ldots, n-1$, we can get the error bound as 


$$
\sum_{j=0}^{n-1} e_{j} L_{j}^{(\alpha)}(x)+\hat{b}_{0}=\sum_{j=0}^{n} a_{j} L_{j}^{(\alpha)}(x) .
$$

As we can see, in Theorem 1 , the computed result $\hat{b}_{0}$ is an exact value with perturbation $\sum_{j=0}^{n-1} e_{j}^{(\alpha)}(x)$. Assuming $c=\sum_{j=0}^{n-1} e_{j} j_{j}^{(\alpha)}(x)$ and $b_{0}=\sum_{j=0}^{n} a_{j} L_{j}^{(\alpha)}(x)$, we obtain

$$
\hat{b}_{0}+c=b_{0} \text {. }
$$

Therefore, we record the round-off error in each step of Clenshaw algorithm by EFTs, and then compute the perturbation to correct the numerical result $\hat{b}_{0}$. In fact, because $c$ is also a Laguerre series, we can apply Clenshaw algorithm again. We present compensated Clenshaw algorithm ( CompClenshaw) in Algorithm 6.

In order to analyze the error bound of the CompClenshaw algorithm, we show Lemma 1 as follow.

Lemma 1. Let $p(x)=\sum_{i=0}^{n} a_{i} L_{i}^{(\alpha)}(x)$ be a Laguerre series of degree $n, x$ is a floating-point number, $\dot{b}_{n}$ the numerical result of Clenshaw algorithm. Then

$$
\mid \text { Clenshaw }(p, x)-p(x)\left|\leq \gamma_{6 n-2} \sum_{j=0}^{n}\right| a_{j} \mid \tilde{L}_{j}^{(\alpha)}(|x|) .
$$

Lemma 2. Suppose $p(x)=\sum_{i=0}^{n} a_{i} L_{i}^{(\alpha)}(x)$ be a Laguerre series of degree $n$ and $x$ is a floatingpoint number, we assume that $\sigma_{i} \leq \omega_{i}\left(\left|A_{i}\right||x|\left|b_{i+1}\right|+\left|B_{i}\right|\left|b_{i+1}\right|+\left|C_{i+1}\right|\left|b_{i+2}\right|+\left|a_{i}\right|\right)$, where $\omega_{i}$ a real numbers. Then

$$
\sum_{j=0}^{n-1} \sigma_{j} \tilde{L}_{j}^{(\alpha)}(|x|) \leq n \omega_{j}\left(1+\gamma_{6(n-1)}\right) \sum_{j=1}^{n}\left|a_{j}\right| \tilde{L}_{j}^{(\alpha)}(|x|) .
$$

Based on Lemma 2, we can obtain Lemma 3 as follow.

Lemma 3. Suppose $g_{i}^{(l)}=A_{i}^{(l)} x \bar{b}_{i+1}+B_{i}^{(l)} \bar{b}_{i+1}+C_{i \pm 1}^{(l)} \bar{b}_{i+2}, q_{i}^{(m)}=A_{i}^{(m)} x \bar{b}_{i+1}+B_{i}^{(m)} \dot{b}_{i+1}+C_{i+1}^{(m)} \dot{b}_{i+2}$, $s_{i}=\alpha_{i}+\beta_{i}+\xi_{i}+\eta_{i}+\theta_{i}+\zeta_{i}, \bar{s}_{i}=\left|\alpha_{i}\right|+\left|\beta_{i}\right|+\left|\xi_{i}\right|+\left|\eta_{i}\right|+\left|\theta_{i}\right|+\left|\zeta_{i}\right|$ and $\tau_{i}$ is the error of ThreeProd, then we can obtain

$$
\begin{gathered}
\left|\sum_{j=0}^{n} g_{j}^{(l)} L_{j}^{(\alpha)}(x)\right| \leq \gamma_{6 n+1} \sum_{j=0}^{n}\left|a_{j}\right| \tilde{L}_{j}^{(\alpha)}(|x|), \\
\left|\sum_{j=0}^{n} g_{j}^{(m)} L_{j}^{(\alpha)}(x)\right| \leq u \gamma_{6 n+1} \sum_{j=0}^{n}\left|a_{j}\right| \tilde{L}_{j}^{(\alpha)}(|x|), \\
\left|\sum_{j=0}^{n-1} S_{j} \tilde{L}_{j}^{(\alpha)}(x)\right| \leq \gamma_{6 n-1} \sum_{j=0}^{n}\left|a_{j}\right| \mid \tilde{L}_{j}^{(\alpha)}(|x|), \\
\left|\sum_{j=0}^{n-1}\right| \tau_{j}\left|\tilde{L}_{j}^{(\alpha)}(x)\right| \leq \gamma_{6} \gamma_{6 n-4} \sum_{j=0}^{n}\left|a_{j}\right| \tilde{L}_{j}^{(\alpha)}(|x|) .
\end{gathered}
$$

As analyzing in Lemma 3, we discover some perturbation do not influence the numerical result in working precision. Such as (16),(18), the bound $u \gamma_{\hbar n+1}, \gamma_{\hbar} \gamma_{\hbar n-4}$ are $O\left(u^{2}\right)$, so these errors do not affect the precision. However, from (15),(17) we know that $\gamma_{\hbar n+1}, \gamma_{h m-1}$ are $O(u)$, so the coefficient perturbations may influence the accuracy, we need to consider it in our error analysis.

Theorem 2. Let $p(x)=\sum_{i=0}^{n} a_{i} L_{i}^{\alpha}(x)$ be a Laguerre series of degree $n,(n \geq 2)$ and $x$ is a floatingpoint number. Then the forward error bound of the CompClenshaw algorithm is 


$$
\mid \text { CompClenshaw }(p, x)-p(x)|\leq u| p(x)\left|+2 \gamma_{6 n}^{2} \sum_{j=0}^{n}\right| a_{j} \mid \tilde{L}_{j}^{(\alpha)}(|x|) \text {. }
$$

Proof. From [9, 15], we show two inequations to get the error bound.

Suppose

then we have

$$
s_{j}=\alpha_{j}+\beta_{j}+\xi_{j}+\eta_{j}+\theta_{j}+\zeta_{j},
$$

Considering that

$$
\hat{s}_{j} \leq\left(1+\gamma_{5}\right)\left(\left|\alpha_{j}\right|+\left|\beta_{j}\right|+\left|\xi_{j}\right|+\left|\eta_{j}\right|+\left|\theta_{j}\right|+\left|\zeta_{j}\right|\right) .
$$

$$
\begin{aligned}
& \left|\sum_{j=0}^{n-1} S_{j} L_{j}^{(\alpha)}(x)-\oplus \sum_{j=0}^{n-1} \hat{s}_{j} \otimes L_{j}^{(\alpha)}(x)\right| \\
& \leq\left|\sum_{j=0}^{n-1} s_{j} L_{j}^{(\alpha)}(x)-\sum_{j=0}^{n-1} \hat{s}_{j} L_{j}^{(\alpha)}(x)\right|+\left|\sum_{j=0}^{n-1} \hat{s}_{j} L_{j}^{(\alpha)}(x)-\oplus \sum_{j=0}^{n-1} \hat{s}_{j} \otimes L_{j}^{(\alpha)}(x)\right| \\
& \leq \sum_{j=0}^{n-1}\left|s_{j}-\hat{s}_{j}\right| L_{j}^{(\alpha)}(|x|)+\gamma_{6 n-7} \sum_{j=0}^{n-1}\left|\hat{s}_{j}\right| \tilde{L}_{j}^{(\alpha)}(|x|) \\
& \leq \gamma_{6 n-2} \sum_{j=0}^{n-1}\left(\left|\alpha_{j}\right|+\left|\beta_{j}\right|+\left|\xi_{j}\right|+\left|\eta_{j}\right|+\left|\theta_{j}\right|+\left|\zeta_{j}\right|\right) \tilde{L}_{j}^{(\alpha)}(|x|) \\
& \leq \gamma_{6 n-2} \gamma_{6 n-1} \sum_{j=0}^{n}\left|a_{j}\right| \tilde{L}_{j}^{(\alpha)}(|x|) \quad[\text { By inequation }(17)] \\
& \leq \gamma_{6 n-1}^{2} \sum_{j=0}^{n}\left|a_{j}\right| L_{j}^{(\alpha)}(|x|)
\end{aligned}
$$

and

$$
\begin{aligned}
& \left|\sum_{j=0}^{n-1} g_{j}^{(l)} L_{j}^{(\alpha)}(x)-\oplus \sum_{j=0}^{n-1} \hat{g}_{j}^{(l)} \otimes L_{j}^{(\alpha)}(x)\right| \\
& \leq\left|\sum_{j=0}^{n-1} g_{j}^{(l)} L_{j}^{(\alpha)}(x)-\sum_{j=0}^{n-1} \hat{g}_{j}^{(l)} L_{j}^{(\alpha)}(x)\right|+\left|\sum_{j=0}^{n-1} \hat{g}_{j}^{(l)} L_{j}^{(\alpha)}(x)-\oplus \sum_{j=0}^{n-1} \hat{g}_{j}^{(l)} \otimes L_{j}^{(\alpha)}(x)\right| \\
& \leq \sum_{j=0}^{n-1}\left|g_{j}^{(l)}-\hat{g}_{j}^{(l)}\right| \tilde{L}_{j}^{(\alpha)}(|x|)+\gamma_{6 n-7} \sum_{j=0}^{n-1}\left|\hat{g}_{j}^{(l)}\right| \tilde{L}_{j}^{(\alpha)}(|x|) \\
& \leq \gamma_{6 n-2} \sum_{j=0}^{n-1}\left(\left|A_{j}^{(l)} x \hat{b}_{j+1}\right|+\left|B_{j}^{(l)} \hat{b}_{j+1}\right|+\left|C_{j+1}^{(l)} \hat{b}_{j+2}\right|\right),
\end{aligned}
$$

combining with

we have

$$
\left|A_{j}^{(l)} x \hat{b}_{j+1}\right|+\left|B_{j}^{(l)} \hat{b}_{j+1}\right|+\left|C_{j+1}^{(l)} \hat{b}_{j+2}\right| \leq u\left(1+\gamma_{1}\right)\left|A_{j} x \hat{b}_{j+1}\right|+\left|B_{j} \hat{b}_{j+1}\right|+\left|C_{j+1} \hat{b}_{j+2}\right|,
$$




$$
\begin{aligned}
& \left|\sum_{j=0}^{n-1} g_{j}^{(l)} L_{j}^{(\alpha)}(x)-\oplus \sum_{j=0}^{n-1} \hat{g}_{j}^{(l)} \otimes L_{j}^{(\alpha)}(x)\right| \\
& \leq \gamma_{6 n-2} u\left(1+\gamma_{1}\right) n\left(1+\gamma_{6(n-1)}\right) \sum_{j=0}^{n}\left|a_{j}\right| \tilde{L}_{j}^{(\alpha)}(|x|) \\
& \leq \gamma_{6 n-2} \gamma_{6 n} \sum_{j=0}^{n}\left|a_{j}\right| \tilde{L}_{j}^{(\alpha)}(|x|) \\
& \leq \gamma_{6 n-1}^{2} \sum_{j=0}^{n}\left|a_{j}\right| \tilde{L}_{j}^{(\alpha)}(|x|) .
\end{aligned}
$$

Then we obtain the error bound

Next we consider that

$$
\begin{aligned}
& \mid \text { CompClenshaw }(p, x)-p(x)|=|(1+\varepsilon)\left(\hat{b}_{0}+\varepsilon \hat{b}_{0}\right)-p(x) \mid \\
& =\left|(1+\varepsilon)\left(p(x)-\varepsilon b_{0}+\varepsilon \hat{b}_{0}\right)-p(x)\right| \quad \text { [By Theroem } \\
& \leq u|p(x)|+(1+u)\left|\varepsilon b_{0}-\varepsilon \hat{b}_{0}\right| .
\end{aligned}
$$

$$
\begin{aligned}
& \left|\varepsilon b_{0}-\varepsilon \hat{b}_{0}\right|=\left|\sum_{j=0}^{n-1} s_{j} L_{j}^{(\alpha)}(x)+\sum_{j=0}^{n-1} g_{j}^{(l)} L_{j}^{(\alpha)} L_{j}^{(\alpha)}(x)-\oplus \sum_{j=0}^{n-1} \hat{s}_{j} \otimes L_{j}^{(\alpha)}(x)-\oplus \sum_{j=0}^{n-1} \hat{g}_{j}^{(l)} \otimes L_{j}^{(\alpha)}(x)\right| \\
& \leq\left|\sum_{j=0}^{n-1} S_{j} L_{j}^{(\alpha)}(x)-\oplus \sum_{j=0}^{n-1} \hat{s}_{j} \otimes L_{j}^{(\alpha)}\right|+\left|\sum_{j=0}^{n-1} g_{j}^{(l)} L_{j}^{(\alpha)}(x)-\oplus \sum_{j=0}^{n-1} g_{j}^{(l)} \otimes L_{j}^{(\alpha)}(x)\right| \\
& \leq 2 \gamma_{6 n-1}^{2} \sum_{j=0}^{n-1}\left|a_{j}\right| \tilde{L}_{j}^{(\alpha)}(|x|) .
\end{aligned}
$$

Finally, we obtain

$$
\begin{aligned}
& \mid \text { CompClenshaw }(p, x)-p(x)|\leq u| p(x)\left|+2(1+u) \gamma_{6 n-1}^{2} \sum_{j=0}^{n}\right| a_{j} \mid \tilde{L}_{j}^{(\alpha)}(|x|) \\
& \leq u|p(x)|+2 \gamma_{6 n}^{2} \sum_{j=0}^{n}\left|a_{j}\right| \tilde{L}_{j}^{(\alpha)}(|x|) .
\end{aligned}
$$

Combining Definition 2 and Theorem 2, we present corollary 3.1 as follow. Given $p(x)=\sum_{i=n}^{n} a_{i} L_{i}^{\alpha}$ a Laguerre series of degree $n$ with floating-point coefficients, and $x$ is a floatingpoint number. Then the error bound of CompClenshaw algorithm is

$$
\frac{|\operatorname{CompClenshaw~}(p, x)-p(x)|}{|p(x)|} \leq u+2 \gamma_{6 n}^{2} \operatorname{cond}(p, x) \text {. }
$$

As the Corollary 3.1 show, the relative error bound produced by CompClenshaw algorithm is $\gamma_{6 n}^{2}$ times the condition number of the polynomial and plus an unavoidable summand $u$. That is to say, if cond $(p, x) \leq 0.5 \gamma_{-2 n}^{-2} \times O(u)$, the computed result of CompClenshaw algorithm is accurate. When cond $(p, x) \geq 0.5 \gamma_{6 n}^{-2^{n}} \times O(u)$, its accuracy will be the same as computed in original Clenshaw algorithm with normal condition numbers.

\section{Experimental Results}

\subsection{Evaluation of the Polynomial in Laguerre basis}

All our experiments are performed using IEEE-754 double precision. The polynomials in Laguerre basis we consider are floating-point coefficients, and $x$ is a floating point. We use the Symbolic Toolbox in Matlab to obtain exact results for comparisons. 
In our experiment, we consider polynomial $p(x)=(x-0.52)^{7}(x-1)^{10}$, an ill-conditional polynomial in neighborhood of its multiple roots 0.52 and 1 . The Clenshaw and CompClenshaw Algorithm are used to evaluate the Laguerre polynomial basis with parameter $\alpha=0$. For perturbations of Laguerre polynomial, we need to convert the polynomial coefficients to Laguerre basis [17]. We use a Convert algorithm [15] to obtain the coefficients of Laguerre polynomial.

Firstly, we check the polynomial with argument $\alpha=0$ for 400 isometric points in [0.68,1.15], [0.7485, 0.7515], and [0.993,1.007]. We show the absolute error (13) and (19) in Figure 1. From Figure 1, we detect that the CompClenshaw algorithm is more accurate than Clenshaw.

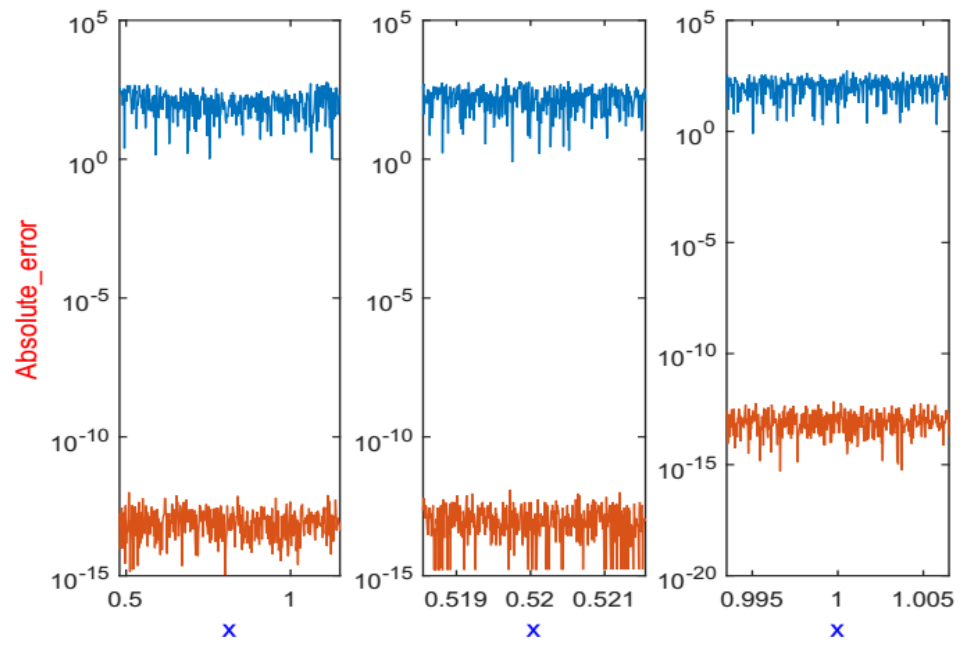

Fig. 1. Absolute error of polynormial from $p(x)=(x-0.75)^{7}(x-1)^{10}$ by the Convert algorithm in Laguerre basis in neighborhood of its multiple roots.using (13) [upper line], the (19) [downward line].

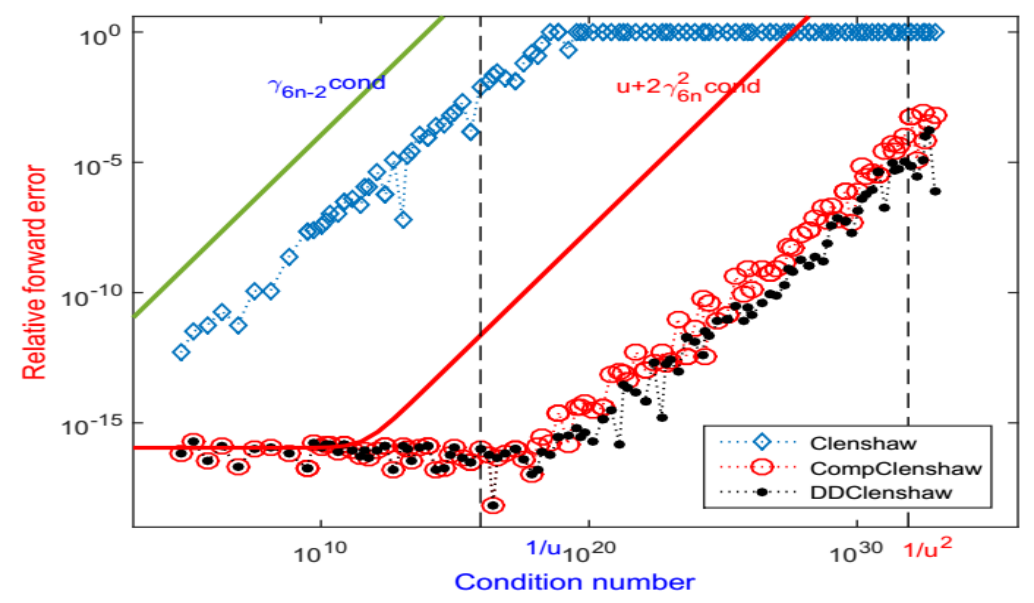

Fig. 2. Forward error of polynomial $p(x)=(x-0.52)^{7}(x-1)^{10}$ evaluate by Clenshaw, CompClenshaw and DDClenshaw algorithm.

As we known, the closer to the root, the larger the condition number [10]. We test 120 points of polynomial $p(x)=(x-0.52)^{7}(x-1)^{10}$ near the root $x=0.52$, i.e. $x=0.52-1.03^{2 i-85}$ where $i=1: 80$ and $x=1-1.03^{5 i-85}$ where $i=1: 40$. We compare the forward relative error $\left|p_{r s s}(x)-p_{s m m}(x)\right| / p_{s m}(x)$ of Clenshaw, CompClenshaw and DDClenshaw algorithm in Figure 2, respectively. As we can see, in Figure 2, the relative error of CompClenshaw and DDClenshaw algorithm are almost same. Moreover, when condition number is less than working precision $u\left(u=1.16 \times 10^{-16}\right)$, the result of 
CompClenshaw is nearly equal to $u$. When condition number is larger than $u$, the forward error increase linearly.

Meanwhile, we also consider the computational complexity of Clenshaw, CompClenshaw, and DDClenshaw algorithms, based on the computation cost of TwoSum, TwoProd, and FastTwoSum. The complexity of them is $7 n-2$ flops, $105 n-9$ flops, $154 n-44$ flops. So we can observe that CompClenshaw is as accurate as DDClenshaw in double precision, but it only needs about $68.18 \%$ of flops.

\section{Conclusion}

This paper introduce a CompClenshaw algorithm to evaluate the Laguerre polynomial. The Clenshaw algorithm is not accurate enough when the problem is ill-conditioned, particularly the floating-point is near the multiple root of polynomial. The CompClenshaw algorithm can delay the appearance of instability problems in standard precision and obtain a full precision result, which as same as computed in double-double precision. Besides, the CompClenshaw algorithm is more efficient to evaluate Laguerre polynomial.

\section{Acknowledgements}

Partially supported by National Natural Science Foundation of China (No. 61571008). Partially supported by National Natural Science Foundation of China (No. 61402495, No. 61602166, No. 61303189, No. 61402496). Partially supported by Science Project of National University of Defense Technology (JC120201) and National Natural Science Foundation of Hunan Province in China (13JJ2001).

\section{References}

[1] C. Hwang and Y. P. Shih. Parameter identification via laguerre polynomials. International Journal of Systems Science, 13(2):209-217, 1982.

[2] M. Gülsu, B. Gürbüz, Y. Öztürk, and M. Sezer. Laguerre polynomial approach for solving linear delay difference equations. Applied Mathematics and Computation, 217(15):6765-6776, 2011.

[3] R. Piessens. Numerical inversion of the laplace transform using generalised laguerre polynomials. Proceedings of the Institution of Electrical Engineers, 118(10):1517-1522, 1971. New York, 19852:309, 1985.

[4] M. W. Coffey. Generalized raising and lowering operators for supersymmetric quantum mechanics. Physics, 2015.

[5] C. W. Clenshaw. A note on the summation of chebyshev series. Mathematics of Computation, 9(51):118-120, 1955.

[6] A. Smoktunowicz. Backward stability of clenshaw's algorithm. BIT Numerical Mathematics, 42(3):600-610, 2002.

[7] R. Barrio. Rounding error bounds for the clenshaw and forsythe algorithms for the evaluation of orthogonal polynomial series. Journal of computational and applied mathematics, 138(2):185-204, 2002.

[8] H. Jiang, R. Barrio, H. Li, X. Liao, L. Cheng, and F. Su. Accurate evaluation of a polynomial in chebyshev form. Applied Mathematics and Computation, 217(23):9702-9716, 2011.

[9] H. Jiang, S. Graillat, C. Hu, S. Li, X. Liao, L. Cheng, and F. Su. Accurate evaluation of the k-th derivative of a polynomial and its application. Journal of Computational and Applied Mathematics, 243:28-47, 2013.

[10] P. Langlois, S. Graillat, and N. Louvet. Compensated horner scheme. 2005.

[11] T. Ogita, S. M. Rump, and S. Oishi. Accurate sum and dot product. SIAM Journal on Scientific Computing, 26(6):1955-1988, 2005.

[12] IEEE. IEEE standard for binary floating-point arithmetic. 1985.

[13] D. E. Knuth. The art of computer programming. Addison-Wesley Pub. Co., 1973. [13] D. E. Knuth. The art of computer programming. Addison-Wesley Pub. Co., 1973.

[14] T. J. Dekker. A floating-point technique for extending the available precision. Numerische Mathematik, 18(3):224-242, 1970.

[15] P. Du, H. Jiang, and L. Cheng. Accurate evaluation of polynomials in legendre basis. Journal of Applied Mathematics, 2014(1):1-13, 2014. 
[16] R. Barrio H. Jiang and S. Serrano. A general condition number for polynomials. SIAM Journal on Numerical Analysis, 51(2):1280-1294, 2013.

[17] R. Barrio and J. M. Peña. Basis conversions among univariate polynomial representations. Comptes Rendus Mathematique, 339(4):293-298, 2004.

[18] S. R. Graillat. Accurate floating-point product and exponentiation. IEEE Transactions on Computers, 58(7):994-1000, 2009.

[19] D. H. Bailey High-precision software directory: QD library, double-double library. Lawrence Berkeley National Laboratory, http://www. nersc. gov/ dhbailey/ mpdist/ mpdist. html. , 2008.

\section{Appendix}

\section{Appendix A: The Extra Proof}

Proof of Theorem 1. Inspired by [15], we set

$$
\begin{aligned}
& s_{j}=\tau_{j}+\alpha_{j}+\beta_{j}+\xi_{j}+\eta_{j}+\theta_{j}+\zeta_{j}, \\
& t_{j}=\left(A_{j}^{(l)}+A_{j}^{(m)}\right) x \hat{b}_{j+1}+\left(B_{j}^{(l)}+B_{j}^{(m)}\right) \hat{b}_{j+1}+\left(C_{j+1}^{(l)}+C_{j+1}^{(m)}\right) \hat{b}_{j+2} .
\end{aligned}
$$

Then

$$
\begin{aligned}
& \hat{b}_{j}+e_{j}=\left(\hat{b}_{j}+s_{j}\right)+t_{j} \\
& =\left(A_{j}^{(h)} x+B_{j}^{(h)}\right) \hat{b}_{j+1}+C_{j+1}^{(h)} \hat{b}_{j+2}+a_{j}+t_{j} \\
& =A_{j} x \hat{b}_{j+1}+B_{j} \hat{b}_{j+1}+C_{j+1} \hat{b}_{j+2}+a_{j}, \quad j=1, \ldots, n,
\end{aligned}
$$

and $\hat{b}_{0}+e_{0}=-x \hat{b}_{1}+\alpha \hat{b}_{1}+\alpha \hat{b}_{2}+a_{0}$.

Thus

$$
\hat{b}_{0}=\sum_{j=0}^{n}\left(a_{j}-e_{j}\right) L_{j}^{(\alpha)}(x)
$$

with $\alpha_{n}=\beta_{n}=\xi_{n}=\eta_{n}=\theta_{n}=\zeta_{n}=0$, then we can obtain the result.

Proof of Lemma 1. Based on equation (3), we obtain

$$
\begin{aligned}
& \hat{b}_{n-1}=A_{n-1}^{(h)} \otimes x \otimes \hat{b}_{n} \oplus B_{n-1}^{(h)} \otimes \hat{b}_{n} \oplus \hat{a}_{j}^{(h)} \\
& =A_{n-1} x \hat{b}_{n}<5>+B_{n-1} \hat{b}_{n}<4>+a_{n-1}<1> \\
& \hat{b}_{n-2}=\left[A_{n-2}^{(h)} \otimes x \oplus B_{n-2}^{(h)}\right] \otimes \hat{b}_{n-1} \oplus C_{n-1}^{(h)} \otimes \hat{b}_{n} \oplus a_{n-2}^{(h)} \\
& =A_{n-2} x \hat{b}_{n-1}<6>+B_{n-2} \hat{b}_{n-1}<5>+C_{n-1} \hat{b}_{n}<4>+a_{n-2}<1>
\end{aligned}
$$

Using mathmematical induction, we can obtain $\hat{b}_{0}$ as follow

$$
\begin{aligned}
& \hat{b}_{0}=<10+6(n-2)>\left(\prod_{j=0}^{n-1} A_{j}\right) x^{n} a_{n}+\cdots+a_{0}<1> \\
& =<6 n-2>\left(\prod_{j=0}^{n-1} A_{j}\right) x^{n} a_{n}+\cdots+a_{0}<1>.
\end{aligned}
$$

Combing (24) the forward error is 


$$
\begin{aligned}
& \left|\hat{b}_{0}-p(x)\right|=\left|\theta_{6 n-2}\left(\prod_{j=0}^{n-1} A_{j}\right) x^{n} a_{n}+\cdots+a_{0} \theta_{1}\right| \\
& \leq \gamma_{6 n-2}\left|\left(\prod_{j=0}^{n-1} A_{j}\right)\right|\left|x^{n}\right|\left|a_{n}\right|+\cdots+\left|a_{0}\right| \\
& =\gamma_{6 n-2} \sum_{j=0}^{n}\left|a_{j}\right| \tilde{L}_{j}^{(\alpha)}(|x|) .
\end{aligned}
$$

Proof of Lemma 2. According to (23), we have

$$
\left|\hat{b}_{j}\right| \leq\left(1+\gamma_{6}\right)\left(\left|A_{j}\|x\| \hat{b}_{j+1}\right|+\left|B_{j}\left\|\hat{b}_{j+1}|+| C_{j+1}\right\| \hat{b}_{j+2}\right|+\left|a_{j}\right|\right) \text {. }
$$

Combining (26) with $\sigma_{j} \leq \omega_{j}\left(A_{j}\left|x\left\|\hat{b}_{j+1}|+| B_{j}\right\| \hat{b}_{j+1}\right|+\left|C_{j+1} \| \hat{b}_{j+2}\right|+\left|a_{j}\right|\right)$, we get

$$
\sigma_{j} \leq \omega_{j}\left(1+\gamma_{6(n-j-1)}\right) \sum_{k=j}^{n}\left|a_{k}\right| \tilde{L}_{k-j}^{(\alpha)}(|x|)
$$

then

$$
\sum_{j=0}^{n-1} \sigma_{j} \tilde{L}_{j}^{(\alpha)}(|x|) \leq \omega_{j}\left(1+\gamma_{6(n-1)}\right) \times \sum_{j=0}^{n-1}\left(\sum_{k=j}^{n}\left|a_{k}\right| \tilde{L}_{k-j}^{(\alpha)}(|x|)\right) \tilde{L}_{j}^{(\alpha)}(|x|) .
$$

Then we can obtain

$$
\sum_{j=0}^{n-1} \sigma_{j} \tilde{L}_{j}^{(\alpha)}(|x|) \leq \omega_{j}\left(1+\gamma_{6(n-1)}\right) \sum_{j=0}^{n-1} \sum_{k=j}^{n}\left|a_{k}\right| \tilde{L}_{k}^{(\alpha)}(|x|) \leq n \omega_{j}\left(1+\gamma_{6(n-1)} \sum_{j=0}^{n-1}\left|a_{j}\right| \tilde{L}_{j}^{(\alpha)}(|x|)\right) .
$$

Proof of Lemma 3. According to $A^{(l)} \leq u A^{(h)}$ and $A^{(h)}=A<1>$,we obtain

$$
\left|g_{j}^{(l)}\right| \leq u\left(1+\gamma_{1}\right)\left(\left|A_{j}\|x\| \hat{b}_{j+1}\right|+\left|B_{j}\left\|\hat{b}_{j+1}|+| C_{j+1}\right\| \hat{b}_{j+2}\right|\right) \text {. }
$$

Then combing Lemma 2

$$
\begin{aligned}
& \left|\sum_{j=0}^{n} g_{j}^{(l)} L_{j}^{(\alpha)}(x)\right| \leq(n+1) u\left(1+\gamma_{6 n+1}\right) \sum_{j=0}^{n}\left|a_{j}\right| \tilde{L}_{j}^{(\alpha)}(|x|) \\
& \leq(6 n+1) u\left(1+\gamma_{6 n+1}\right) \sum_{j=0}^{n}\left|a_{j}\right| \tilde{L}_{j}^{(\alpha)}(|x|) \\
& \leq \gamma_{6 n+1} \sum_{j=0}^{n}\left|a_{j}\right| \tilde{L}_{j}^{(\alpha)}(|x|) .
\end{aligned}
$$

Similarly, we have

$$
\left|g_{j}^{(m)}\right| \leq u^{2}\left(1+\gamma_{1}\right)\left(\left|A_{j}\|x\| \hat{b}_{j+1}+\right| B_{j}\|x\| \hat{b}_{j+1}|+| C_{j+1} \| \hat{b}_{j+2} \mid\right) .
$$

Then

$$
\left|\sum_{j=0}^{n} g_{j}^{(m)} L_{j}^{(\alpha)}(x)\right| \leq u \gamma_{6 n+1} \sum_{j=0}^{n}\left|a_{j}\right| \tilde{L}_{j}^{(\alpha)}(|x|)
$$

Based on equation (4), we obtain 


$$
\begin{aligned}
& \left|\alpha_{j}\right| \leq\left(2 u+14 u^{2}\right)\left(1+\gamma_{1}\right)\left|A_{j} x \hat{b}_{j+1}\right|, \\
& \left|\beta_{j}\right| \leq u\left|B_{j}^{(h)} \hat{b}_{j+1}\right| \leq u\left(1+\gamma_{1}\right)\left|B_{j} \hat{b}_{j+1}\right|, \\
& \left|\xi_{j}\right| \leq u\left|C_{j+1}^{(h)} \hat{b}_{j+2}\right| \leq u\left(1+\gamma_{1}\right)\left|C_{j+1} \hat{b}_{j+2}\right|, \\
& \left|\eta_{j}\right| \leq u\left|s_{j}+t_{j}\right| \leq u\left|A_{j} x \hat{b}_{j+1}<3>+B_{j} \hat{b}_{j+1}<2>\right|, \\
& \left|\theta_{j}\right| \leq u\left|A_{j} x \hat{b}_{j+1}<4>+B_{j} \hat{b}_{j+1}<3>+C_{j+1} \hat{b}_{j+2}<2>\right|, \\
& \left|\zeta_{j}\right| \leq u\left|A_{j} x \hat{b}_{j+1}<5>+B_{j} \hat{b}_{j+1}<4>+C_{j+1} \hat{b}_{j+2}<3>+a_{j}<1>\right|,
\end{aligned}
$$

Then

$$
\bar{s}_{j} \leq\left(5 u+14 u^{2}\right)\left|1+\gamma_{5}\right| \times\left(\left|A_{j} x \| \hat{b}_{j+1}+\right| B_{j} \hat{b}_{j+1}|+| C_{j+1} \hat{b}_{j+2}|+| a_{j} \mid\right) .
$$

Let $\omega_{j}=\left(5 u+14 u^{2}\right)\left|1+\gamma_{5}\right|$, from Lemma 2 we have

$$
\begin{aligned}
& \left|\sum_{j=0}^{n-1}\left(\left|\alpha_{j}\right|+\left|\beta_{j}\right|+\left|\xi_{j}\right|+\left|\eta_{j}\right|+\left|\theta_{j}\right|+\left|\zeta_{j}\right|\right) L_{j}^{(\alpha)}(x)\right| \\
& \leq(5+14 u) n u\left(1+\gamma_{6 n-1}\right) \sum_{j=0}^{n} \mid a_{j} \| \tilde{L}_{j}^{(\alpha)}(|x|) \\
& \leq \gamma_{6 n-1} \sum_{j=0}^{n} \mid a_{j} \| \tilde{L}_{j}^{(\alpha)}(|x|), \quad(n>1) .
\end{aligned}
$$

Similarly, combing with $\left|\tau_{j}\right| \leq \gamma_{2} \gamma_{6}\left|A_{j}^{(h)} x \hat{b}_{j+1}\right| \leq \gamma_{2} \gamma_{6}\left(1+\gamma_{1}\right)\left|A_{j} x \hat{b}_{j+1}\right|$, we have

$$
\begin{aligned}
& \left|\sum_{j=0}^{n-1}\right| \tau_{j}\left\|L_{j}^{(\alpha)}(x)\left|\leq n \gamma_{2} \gamma_{6}\left(1+\gamma_{1}\right)\left(1+\gamma_{6(n-1)}\right) \sum_{j=0}^{n}\right| a_{j}\right\| L_{j}^{(\alpha)}(|x|) \\
& \leq \gamma_{6} \gamma_{6 n-4} \sum_{j=0}^{n} \mid a_{j} \| \tilde{L}_{j}^{(\alpha)}(|x|) .
\end{aligned}
$$

\section{Appendix B: The double-double Algorithm [19]}

Algorithm 7 Addition of double-double number and a double number

$[r h, r l]=a d d \_d d \_d(a h, a l, b)$

$[t h, t l]=\operatorname{TwoSum}(a h, b)$

$t l=a l \oplus t l$

$[r h, r l]=$ FastTwoSum $(t h, t l)$

Algorithm 8 Multiplication of a double-double number by a double number

$[r h, r l]=$ prod_dd_d $(a h, a l, b)$

$[$ th, $t l]=\operatorname{TwoProd}(a h, b)$

$t l=a l \otimes b \oplus t l$

$[r h, r l]=$ FastTwoSum $($ th,$t l)$

Algorithm 9 Multiplication of a double-double number by a double-double number $[r h, r l]=$ prod_dd_dd $(a h, a l, b h, b l)$

$[t h, t l]=\operatorname{TwoProd}(a h, b h)$

$t l=(a h \otimes b l) \oplus(a l \otimes b h) \oplus t l$

$[r h, r l]=$ FastTwoSum $($ th,$t l)$ 


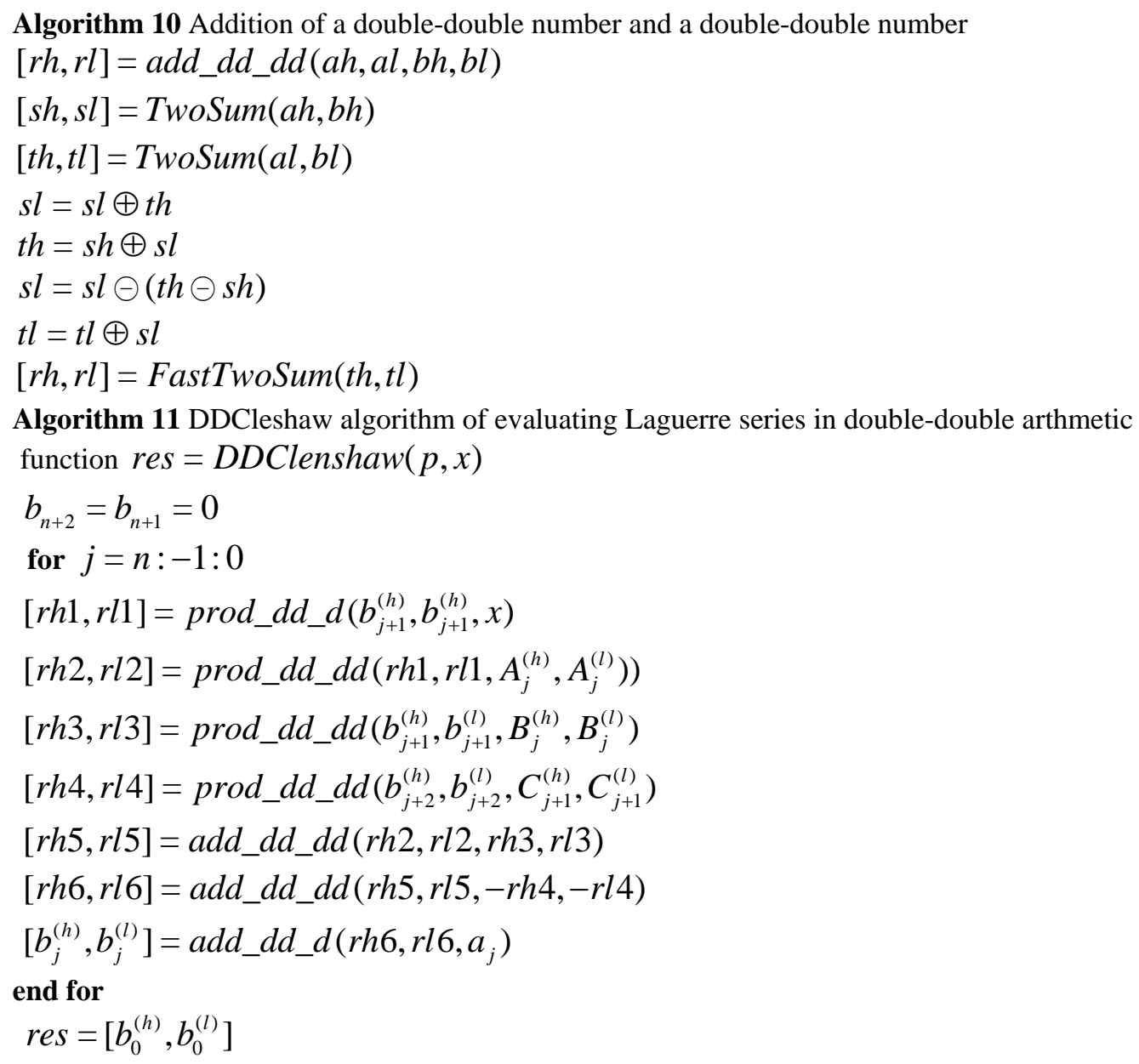

